\title{
Yukawa Textures, Neutrino Masses and Horava-Witten M-Theory
}

\author{
R. Arnowitt,,$*$ B. Dutta, ${ }^{2,+}$ and B. $\mathrm{Hu}^{1, t}$ \\ ${ }^{1}$ Center For Theoretical Physics, Department of Physics, \\ Texas A\&M University, College Station, TX, 77843-4242, USA \\ ${ }^{2}$ Department of Physics, University of Regina, \\ Regina SK, S4S 0A2, Canada
}

(Dated: September 12, 2018)

\begin{abstract}
We consider the Horava-Witten based model with 5-branes situated near the distant orbifold plane and with vanishing instanton numbers on the physical plane. This model has a toric fibered Calabi-Yau with del Pezzo base $d P_{7}$ which allows three generations with Standard Model gauge group at the GUT scale. Previous analysis showed that the quark hierarchy at the electroweak scale could be achieved qualitatively without undue fine tuning due to the effects of the 5-branes on the Kahler potential. We extend here this analysis to include the leptons. A new mechanism is introduced to obtain neutrino masses by assuming massless right handed neutrinos exist in the particle spectrum, which allows a cubic holomorphic term to exist in the Kahler metric, $l_{L} H_{2} \nu_{R}$, scaled by the $11 D$ Planck mass. After transferring this term to the superpotential, this term gives rise to neutrino masses of the correct size at the electroweak scale. With natural choices of the Yukawa and Kahler matrix entries, it is possible to fit all mass, CKM and MNS experimental data. The model predicts $\mu \rightarrow e+\gamma$ decay at a rate that should be detectable for much of the SUSY parameter space in the next round of experiments.
\end{abstract}

\footnotetext{
*Electronic address: arnowitt@physics.tamu.edu

${ }^{\dagger}$ Electronic address: duttabh@yogi.phys.uregina.ca

${ }^{\ddagger}$ Electronic address: b-hu@physics.tamu.edu
} 


\section{INTRODUCTION}

While the Standard Model (SM) has been successful in fitting all current accelerator data, the origin of the quark and lepton mass spectrum remains a puzzle requiring further understanding. Thus the explanation of the striking hierarchy of masses (e. g. the up to top quark mass ratio is $m_{u} / m_{t} \simeq 10^{-5}$ ) and the hierarchy of elements in the CKM matrix all are beyond the scope of the Standard Model. The matter has been further exacerbated by the discovery of neutrino masses, since now in addition there is need for an explanation of the MNS matrix as well as the origin of the very tiny neutrino masses. A large number of suggestions exist in the literature attempting to explain these properties of quarks and leptons. One approach, starting perhaps with the work of Georgi and Jarlskog [1], suggests that the fundamental origin of quark and lepton masses is to be found at high energies, i. e. the GUT scale, $M_{G} \cong 3 \times 10^{16} \mathrm{GeV}$, and the complexity we see at low energies arises from the running of the renormalization group equations (RGEs) down to the electroweak scale. This approach, however, has not appeared to be too promising. Thus a general analysis of the $u$ and $d$ Yukawa matrices with five zeros at the GUT scale given in 2] is shown in Table 1. Here $\lambda=0.2$ is the Wolfenstein parameter, and the choice of Table 1, when evaluated at the electroweak scale does indeed agree approximately with the quark masses and CKM matrix. However, to generate the experimental hierarchy one has to have entries at the GUT scale of size $\lambda^{6} \simeq 10^{-5}$, showing that the problem at the GUT scale is very much the same as at the electroweak scale.

Table 1. Approximate Yukawa textures at $M_{G}$ for $Y_{U}$ and $Y_{D}$ where $\lambda=0.2[2]$.

$$
Y_{U}=\left(\begin{array}{ccc}
0 & \sqrt{2} \lambda^{6} & 0 \\
\sqrt{2} \lambda^{6} & \sqrt{3} \lambda^{4} & \lambda^{2} \\
0 & \lambda^{2} & 1
\end{array}\right) ; Y_{D}=\left(\begin{array}{ccc}
0 & 2 \lambda^{4} & 0 \\
2 \lambda^{4} & 2 \lambda^{3} & 0 \\
0 & 0 & 1
\end{array}\right)
$$

String theory represents at present the only model that has been proposed which in principle can calculate the Yukawa matrices from first principles. Unfortunately, mathematical tools to explicitly do this have not yet been developed. In spite of this, the 
general formulation of the Yukawa problem in string theory opens new windows for seeing how the quark and lepton hierarchies might naturally have arisen, approaches not available in standard SUGRA GUT theory. In particular, the Horava-Witten heterotic M-Theory [3, 4], which offers a natural explanation of why grand unification can occur at $M_{G}$ rather than the Planck scale $M_{P}$, has had significant development 5, 6, 17, 8, 9, 10, 11, 12, 13, 14, 15, 16, 17, 18, 19, 20, 21, 22, 23, 24, 25, 26, 27] giving rise to three generation models with the SM low energy gauge group $S U(3) \times S U(2) \times U(1)$. In this model, physical space is one of two 10 dimensional (10D) orbifold planes separated by a finite distance in the 11 th dimension, the theory obeying $S^{1} / Z_{2}$ symmetry in the 11 th dimension. Six of the 10 dimensions are compactified to a Calabi-Yau (C-Y) threefold, the remaining four being Minkowski space. An array of six dimensional 5-branes perpendicular to the 11th dimension can exist between the two orbifold planes. While it is not possible to make first principle calculations, one can examine whether the general structure of such a theory can replicate the SM at low energy. In this connection, it was seen in [28] that the general structure of the quark mass matrices can arise without undue fine tuning if the 5-branes lie close to the distant orbifold plane, and the instanton number of the physical orbifold plane, $\beta^{(0)}$ vanished. It was explicitly shown in [28] that a three generation model with $\beta^{(0)}=0$ and SM gauge group indeed can exist for a torus fibered Calabi-Yau (with two sections) with del Pezzo base $d P_{7}$. The quark and CKM matrix were calculated for a model of this type in agreement with data, and it was shown also in approximate analytic calculations how the mass hierarchies can arise without undue fine tuning due to the general structure of the Kahler potential.

In this paper we extend the analysis of [28] in two directions. We first include the charged lepton mass matrix and obtain the mass hierarchies experimentally seen. We then consider neutrino masses. The conventional way for accounting for the very small mass of neutrinos is the seesaw mechanism [29] which gives rise to Majorana neutrino masses. We consider here, however, a new way of achieving small neutrino masses based on the structure of the Kahler potential. This mechanism is different from the seesaw mechanism, and gives rise to Dirac masses for the neutrinos. Neutrino masses and the MNS matrix [30] are calculated consistent with the large mixing angle (LMA) analysis of the solar, atmospheric, reactor and long baseline neutrino data (e.g. see [31] for a global analysis in the context of three-neutrino oscillations). Because of the appearance of the MNS matrix, lepton flavor violation processes, 
which are absent in the SM, will occur in this model. SM contributions here are too small to be observed experimentally. However, SUSY contributions are much larger. We have studied the $\mu \rightarrow e+\gamma$ decay in this model including all possible contributions. For large $\tan \beta$, the branching ratio for $\mu \rightarrow e+\gamma$ is close to the current experimental bound [32], and would be accessible to future experiments [33, 34]. A summary of some of the above results was given in [35].

Our paper is organized as follows. In Sec. 2 we give a brief review of M-Theory, and the basic results obtained in [28] for torus fibered Calabi-Yau manifolds. In Sec. 3 we review and update the results of [28] for the quark masses and extend this analysis to the lepton sector. In Sec. 4 we introduce the new mechanism to obtain small neutrino masses and calculate the masses and mixing angles for this model. In Sec. 5 we present our calculation for the $\mu \rightarrow e+\gamma$ decay. Finally, conclusions are given in Sec. 6 .

\section{HORAVA-WITTEN KAHLER POTENTIAL}

The Horava-Witten M-Theory is concerned with 11 dimensional supergravity on an orbifold $M_{10} \times S^{1} / Z_{2}$, where $Z_{2}$ is reflection of the 11 th coordinate. One can think of this space as an 11 dimensional space $M_{11}$ bounded by two 10 dimensional orbifold planes $M_{10}$ at $x^{11}=0$ and $\pi \rho$. In the simplest case, $M_{10}$ is the product space $M_{4} \times X$ where $M_{4}$ is Minkowski space and $X$ is a (compact) C-Y threefold, the physical world living on one of the orbifold planes (e.g. $x^{11}=0$ ), the other orbifold plane being a "hidden" sector. In addition, there may be six dimensional 5 -branes lying along $x^{11}$ at bulk points $x_{n}$ with $0<x_{n}<\pi \rho$, parallel to the orbifold planes, each with four dimensions spanning $M_{4}$, the additional two dimensions wrapped around a holomorphic curve in the Calabi-Yau space. The construction of a consistent theory involves a remarkable set of interlocking constraints due to anomaly cancellation, gauge invariance, and local supersymmetry leading naturally to a theory which possesses a number of properties appropriate for phenomenology. Thus there must be $E_{8}$ gauge interactions with chiral multiplets on each $M_{10}$ orbifold plane (SO(32) being excluded) which can easily be broken on the physical plane to the SM group by Wilson lines. The 10D gauge coupling constant, $\lambda$, is uniquely determined in terms of the 11D Planck mass, $\kappa^{-2 / 9}$, leading to the result that the fundamental scale of nature, the $11 \mathrm{D}$ Planck mass, is $\mathcal{O}\left(M_{G}\right)$, and explaining why grand unification occurs at $M_{G}$ rather than the $4 \mathrm{D}$ Planck mass (which 
is a derived quantity). Finally, a consistent theory exists only as a quantum theory (the classical theory being inconsistent), something one would hope might be true for any fundamental theory. Much progress has been made in showing what the low energy structure of such a theory might be, and models with three generations of quarks and leptons obeying the SM gauge group have been constructed. While the details of the construction of the theory given in [3, 4] is rather intricate, it is possible to see how the different elements interact to produce a physically interesting model and so we first briefly summarize this construction. We then give the relevant formulae needed to examine the low energy structure. Details of the latter can be found in [20], and for the specific model considered here in [28].

The field content of $11 \mathrm{D}$ supergravity is the metric $g_{I J}$, the gravitino $\psi_{I J}$, the three form $C_{I J K}$ and its field strength $G_{I J K L}$. (In lowest order $G_{I J K L}=d_{I} C_{J K L}$.) The Bose part of the Lagrangian is :

$$
\begin{aligned}
L_{S}= & \frac{1}{\kappa^{2}} \int_{M^{11}} d^{11} x \sqrt{g}\left(-\frac{1}{2} R-\frac{1}{48} G_{I J K L} G^{I J K L}\right. \\
& \left.-\frac{\sqrt{2}}{3456} \epsilon^{I_{1} I_{2} \ldots I_{11}} C_{I_{1} I_{2} I_{3}} G_{I_{4} \ldots I_{7}} G_{I_{8} \ldots I_{11}}\right)
\end{aligned}
$$

where the field strengths obey the field equations $D^{I} G_{I J K L}=0$, and the Bianchi identity $d G_{I J K L M}=0$. Here $\kappa$ is the $11 \mathrm{D}$ gravitational constant. The Horava-Witten theory comes about as follows. While in a smooth manifold 11D supergravity has no anomalies, on an orbifold anomalies arise at the fixed points $x=0$ and $x=\pi \rho$. To cancel these, it is necessary to put Yang Mills multiplets on each $M_{10}$ orbifold plane, and the cancellation occurs only if the gauge group on each manifold is (the phenomenologically desirable) $E_{8}$. To lowest order, the Yang Mills Lagrangian on each $M_{10}$ reads then:

$$
L_{Y M}=-\frac{1}{\lambda^{2}} \int_{M^{10}} d^{10} x \sqrt{g} \operatorname{tr}\left(\frac{1}{4} F_{A B} F^{A B}+\frac{1}{2} \bar{\chi} \Gamma^{A} D_{A} \chi\right) .
$$

where $A, B=1,2 \ldots 10$, and $\chi$ is the associated gaugino. However, Eq. (2) is not locally supersymmetric, and one must proceed in the usual fashion to add additional interactions and modifications of the transformation laws to achieve local supersymmetry. As usual, this involves coupling the gravitino to the Yang Mills supercurrent. However, unlike the case where the Yang Mills and supergravity multiplets live in the same space, the gravitino here lives in the 11D bulk, while the Yang Mills multiplet is constrained to live in 10D. For this situation, a locally supersymmetric Yang Mills theory cannot be achieved simply by 
adding interactions on the orbifold plane. It turns out that a supersymmetric theory can be achieved only by modifying the Bianchi identities to read

$$
d G_{11 A B C D}=8 \pi^{2} \sqrt{2} \frac{\kappa^{2}}{\lambda^{2}} \Sigma_{0}^{N+1} J^{(n)} \delta\left(x^{11}-x_{n}\right) .
$$

where $x_{0}=0, x_{N+1}=\pi \rho$ and $x_{n}, n=1 \ldots N$ are the positions of the five branes,

$$
J^{(0, N+1)}=-\frac{1}{16 \pi^{2}}\left(\operatorname{tr} F \wedge F-\frac{1}{2} \operatorname{tr} R \wedge R\right)_{x^{11}=0, \pi \rho} .
$$

and $J^{(n)}, n=1 \ldots N$ are sources from the 5-branes. With Eq.(3), the total supergravity + (modified) Yang Mills Lagrangian can be made locally supersymmetric. However, having gained supersymmetry, one has lost Yang Mills gauge invariance (!). For while Eq.(3) implies that $G_{A B C D}$ is gauge invariant, the corresponding potential $C_{11 A B}$ now is not, i.e. under a Yang Mills gauge transformation one has

$$
\delta C_{11 A B}=-\frac{\kappa^{2}}{6 \sqrt{2} \lambda^{2}}\left[\operatorname{tr}\left(\epsilon F_{A B} \delta\left(x^{11}\right)\right)+\operatorname{tr}\left(\epsilon F_{A B} \delta\left(x^{11}-\pi \rho\right)\right)\right] .
$$

which implies the $C \wedge G \wedge G$ term of Eq.(11) is not gauge invariant. Thus the classical theory is not gauge invariant, and a consistent classical theory does not exist. However, in the quantum theory, there is in addition the 10D Majorana-Weyl anomaly, and due to unique features of the $E_{8}$ group (!) can cancel the loss of gauge invariance of the "Green-Schwarz" $C \wedge G \wedge G$ term provided

$$
\lambda^{2}=2 \pi(4 \pi \kappa)^{2 / 3}
$$

Thus only a consistent quantum theory can be built, and this quantum theory determines the $10 \mathrm{D}$ gauge coupling constant in terms of the 11D gravitational constant.

Eq.(6) leads immediately to interesting phenomenological consequences. For compactifying $M_{11}$ on a Calabi-Yau manifold, one has to lowest order for the 4D gauge coupling constant and Newton constant [5]

$$
\alpha_{G}=\frac{\left(4 \pi \kappa^{2}\right)^{2 / 3}}{2 \mathcal{V}} ; \quad G_{N}=\frac{\kappa^{2}}{16 \pi^{2} \mathcal{V} \rho}
$$

where $\mathcal{V}$ is the Calabi-Yau volume. Setting $\mathcal{V}^{1 / 6}=1 / M_{G}$ (so that grand unification occurs at the compactification scale as required by the LEP data) and using $\alpha_{G}=1 / 24$, one finds that the fundamental 11D Planck mass is $\kappa^{-2 / 9} \cong 2 M_{G}$ and $\pi \rho^{-1} \cong 4.7 \times 10^{15} \mathrm{GeV}$. Alternately one may say that the 11D Planck mass is the fundamental scale and it sets the GUT scale, 
while the largeness of the $4 \mathrm{D}$ Planck mass is due mostly to accidental $4 \pi$ factors arising in the analysis.

We now summarize the basic formulae of [20] and 28] needed to build a phenomenologically acceptable theory. The sources $J^{(n)}$ of Eq. (3) play an important role in building a model. Thus if integrated over a set of independent 4 cycles $C_{4 i}$, they define integer charges:

$$
\beta_{i}^{(n)}=\int_{C_{4 i}} J^{(n)}
$$

and Eq.(3) then implies $\Sigma \beta_{i}^{(n)}=0$. Here $\beta_{i}^{(0)}$ and $\beta_{i}^{(N+1)}$ are the instanton charges on the orbifold planes and $\beta_{i}^{(n)}(n=1 \ldots N)$ are the magnetic charges of the 5 -branes. The existence of non-zero instanton Yang Mills fields with gauge group G on the orbifold plane implies that $E_{8}$ breaks into $G \times H$ where $\mathrm{H}$ is the remaining symmetry at the GUT scale of the physical theory. We chose here $G=S U(5)$ so that $H=S U(5)^{1}$.

Chiral matter arises from the components of the Yang Mills multiplet in the Calabi-Yau part of the $M_{10}$ orbifold [20]. Thus labeling the C-Y indices by holomorphic (anti-holomorphic) coordinates $a(\bar{a})=1,2,3$, then one can expand e.g. $F_{\mu \bar{b}}$ in terms of a basis set of functions $u_{I}^{x}$ in the $\mathrm{C}-\mathrm{Y}$ space ( $\mathrm{I}$ is a family index and $\mathrm{x}$ a representation index), the coefficients in the Minkowski space being the scalar components of the chiral multiplets $C(R)^{I p}$ (where $\mathrm{R}$ is the representation):

$$
F_{\mu \bar{b}}=\sqrt{2 \pi \alpha_{G}} \sum_{R} u_{I \bar{b}}^{x}(R) T_{x p}(R)\left(D_{\mu} C(R)\right)^{I p} .
$$

In terms of these quantities, one then defines the metric

$$
G_{I J}\left(a^{i} ; R\right)=\frac{1}{v V} \int_{X} \sqrt{g} g^{a \bar{b}} u_{I a x}(R) u_{J \bar{b}}^{x}(R)
$$

and the Yukawa couplings [20]

$$
\lambda_{I J K}\left(R_{1}, R_{2}, R_{3}\right)=\int_{X} \Omega \wedge u_{I}^{x}\left(R_{1}\right) \wedge u_{J}^{y}\left(R_{2}\right) \wedge u_{K}^{z}\left(R_{3}\right) f_{x, y, z}^{\left(R_{1}, R_{2}, R_{3}\right)}
$$

where $\Omega$ is the covariantly constant $(3,0)$ form, f projects out the gauge singlet parts, and $\mathcal{V} \equiv v V$ is the volume of the Calabi-Yau space while $v$ is the coordinate volume:

$$
V=\frac{1}{v} \int_{X} d^{6} x \sqrt{g} ; \quad v=\int_{X} d^{6} x
$$

\footnotetext{
${ }^{1}$ Recently, an alternate choice, $G=S U(4)$ and $H=S O(10)$ has been considered by A. E. Faraggi and R. S. Garavuso [36, 37].
} 
In addition one defines the $S, T^{i}$ and 5 -brane moduli by

$$
R e(S)=V ; \operatorname{Re} T^{i}=V^{-1 / 3} R a^{i} ; \operatorname{Re} Z_{n}=z_{n}
$$

where the modulus $R$ is the orbifold radius divided by $\rho$ and $z_{n}=x_{n} / \pi \rho$. $V$ can be expressed in terms of the $a^{i}$ moduli by $V(a)=\frac{1}{6} d_{i j k} a^{i} a^{j} a^{k}$ where $d_{i j k}$ are the Calabi-Yau intersection numbers :

$$
d_{i j k}=\int_{X} \omega_{i} \wedge \omega_{j} \wedge \omega_{k}
$$

Following the techniques of [5], the field equations and Bianchi identities in Eq.(3) were solved in the presence of 5-branes to leading order $O\left(\kappa^{2 / 3}\right)$ [20] leading to an effective four dimensional Lagrangian at compactification scale $M_{G}$. We now state the results that were obtained. The gauge kinetic functions on the orbifold planes are given by

$$
\begin{aligned}
& f^{(1)}=S+\epsilon T^{i}\left(\beta_{i}^{(0)}+\sum_{n=1}^{N}\left(1-Z_{n}\right)^{2} \beta_{i}^{(n)}\right) \\
& f^{(2)}=S+\epsilon T^{i}\left(\beta_{i}^{(N+1)}+\sum_{n=1}^{N} Z_{n}^{2} \beta_{i}^{(n)}\right)
\end{aligned}
$$

where

$$
\epsilon=\left(\frac{\kappa}{4 \pi}\right)^{2 / 3} \frac{2 \pi^{2} \rho}{\mathcal{V}^{2 / 3}}
$$

The matter Kahler potential, $K=Z_{I J} \bar{C}^{I} C^{J}$, on the physical orbifold plane at $x^{11}=0$ has the Kahler metric

$$
Z_{I J}=e^{-K_{T} / 3}\left[G_{I J}-\frac{\epsilon}{2 V} \tilde{\Gamma}_{I J}^{i} \sum_{n=0}^{N+1}\left(1-z_{n}\right)^{2} \beta_{i}^{(n)}\right]
$$

where

$$
\begin{gathered}
K_{T}=-\ln \left[\frac{1}{6} d_{i j k}\left(T^{i}+\bar{T}^{i}\right)\left(T^{j}+\bar{T}^{j}\right)\left(T^{k}+\bar{T}^{k}\right)\right] \\
\tilde{\Gamma}_{I J}^{i}=\Gamma_{I J}^{i}-\left(T^{i}+\bar{T}^{i}\right) G_{I J}-\frac{2}{3}\left(T^{i}+\bar{T}^{i}\right)\left(T^{k}+\bar{T}^{k}\right) K_{T k j} \Gamma_{I J}^{j}
\end{gathered}
$$

and

$$
K_{T i j}=\frac{\partial^{2} K_{T}}{\partial T_{i} \partial \bar{T}^{j}} ; \Gamma_{I J}^{i}=K_{T}^{i j} \frac{\partial G_{I J}}{\partial T^{j}}
$$

The Yukawa matrices are

$$
Y_{I J K}=2 \sqrt{2 \pi \alpha_{G}} \lambda_{I J K} \simeq 1.02 \lambda_{I J K}
$$

for $\alpha_{G}=1 / 24$. The Kahler metric on the distant orbifold plane at $x^{11}=\pi \rho$ is given by Eq.(17) with $z_{n} \rightarrow\left(1-z_{n}\right)$. 


\section{YUKAWA TEXTURES}

The Yukawa couplings are given in Eqs.(11) and (21) as integrals over the C-Y space. A priori there is no reason to suggest that a hierarchy such as Table 1 should arise and one expects that the non-zero entries to be $O(1)$. Similarly, one expects a priori that the non-zero elements of $G_{I J}$ in Eq.(10) be of $O(1)$. However, a mild hierarchy can develop in the Kahler metric of Eq. (17) if the 5-branes all lie close to the distant orbifold plane, i. e. $d_{n}=1-z_{n} \cong 0.1$, and provided also $\beta^{(0)}=0$. Then the second term will be small compared to the first $(\epsilon \cong 0.9)$, and the model of [28] assumed that $G_{I J}$ contributes only to the first two generations of the $u$ quark and $d_{L}$ (which appear together in the $S U(5) 10$ representation) but to all generations of $d_{R}$, while the second term contributes to all generations but is then dominant for the third generation of $u_{L}, u_{R}, d_{L}$. (That a C-Y manifold exits with $\beta^{(0)}=0$ with three generations and a SM gauge group is non-trivial and was explicitly shown to be possible in [28].) When the Kahler metric was diagonalized to a unit matrix, it was seen that this idea was sufficient to generate a satisfactory explanation of the more extreme Yukawa hierarchies at the electroweak scale, and we extend this idea here to the lepton sector. Thus the Kahler metric has the general form

$$
Z^{F}=f_{T}\left(\begin{array}{ccc}
1 & O(1) & O\left(d^{2}\right) \\
O(1) & O(1) & O\left(d^{2}\right) \\
O\left(d^{2}\right) & O\left(d^{2}\right) & O\left(d^{2}\right)
\end{array}\right)
$$

where $\mathrm{F}$ stands for the different matter fields: $q=u_{L}, u_{R}, d_{L}, l=\left(\nu_{L}, e_{L}\right)$ and $e=e_{R}$ and $f_{T}$ is given from Eq.(17) to be $e^{-K_{T} / 3}$. We assume that $G_{I J}$ has non-zero elements of $O(1)$ for all generations of $d_{R}$. (For convenience, we've re-scaled the $Z_{11}^{F}$ entry in Eq. (22) to 1.) The hierarchy then arises when one transforms the $Z_{I J}$ to the unit matrix by a unitary matrix $U$ and a diagonal scaling matrix $S$ to obtain the canonical matter fields $C_{F}^{I^{\prime}}$ :

$$
C_{F}^{I}=\frac{1}{\sqrt{f_{T}}}\left(U^{(F)} S^{(F)}\right)_{I J} C_{F}^{J^{\prime}}
$$

where

$$
\operatorname{diag} S^{(F)}=\left(\lambda_{F 1}^{-1 / 2}, \lambda_{F 2}^{-1 / 2}, \lambda_{F 3}^{-1 / 2}\right)
$$

and $\lambda_{F i}, i=1,2,3$ are the eigenvalues of $Z_{I J}^{F} / f_{T}$. A similar transformation is made on the Higgs fields contribution to the Kahler potential

$$
f_{T} G_{H_{1,2}} \bar{H}_{1,2} H_{1,2}
$$


with rescaling of $H_{1,2}$ :

$$
H_{1,2}=\frac{1}{\sqrt{f_{T} G_{H_{1,2}}}} H_{1,2}^{\prime}
$$

Before making the transformation of Eq. (23), The Yukawa contribution to the superpotential is [20]

$$
W_{Y}=e^{\frac{1}{2} K_{m}} \frac{1}{3} Y_{I J K} C^{I} C^{J} C^{K}
$$

where $K_{m}=\ln (S+\bar{S})+K_{T}$ is the moduli contribution to the Kahler potential. From Eqs. (13) and (18), one has

$$
K_{m}=-\ln (2 V)-\ln \left(8 R^{3}\right) .
$$

Written in terms of SM fields $W_{Y}$ then is

$$
W_{Y}=\frac{1}{4 R^{3 / 2} V^{1 / 2}}\left(Y^{(u)} q_{L} H_{2} u_{R}+Y^{(d)} q_{L} H_{1} d_{R}+Y^{(e)} l_{L} H_{1} e_{R}\right) .
$$

and after the transformation to the canonical matter fields one has

$$
W_{Y}=u_{L}^{\prime} \lambda^{(u)} u_{R}^{\prime} H_{2}^{\prime}+d_{L}^{\prime} \lambda^{(d)} d_{R}^{\prime} H_{1}^{\prime}+e_{L}^{\prime} \lambda^{(e)} e_{R}^{\prime} H_{1}^{\prime}
$$

where $\lambda^{(u, d, e)}$ are give by ${ }^{2}$

$$
\begin{aligned}
& \lambda_{I J}^{(u)}=\frac{1}{8 \sqrt{2}} \frac{1}{R^{3} V^{1 / 2}} \frac{1}{\sqrt{G_{H_{2}}}}\left(S^{(q)} \tilde{U}^{(q)} Y^{(u)} U^{(u)} S^{(u)}\right)_{I J} \\
& \lambda_{I J}^{(d)}=\frac{1}{8 \sqrt{2}} \frac{1}{R^{3} V^{1 / 2}} \frac{1}{\sqrt{G_{H_{1}}}}\left(S^{(q)} \tilde{U}^{(q)} Y^{(d)} U^{(d)} S^{(d)}\right)_{I J} \\
& \lambda_{I J}^{(e)}=\frac{1}{8 \sqrt{2}} \frac{1}{R^{3} V^{1 / 2}} \frac{1}{\sqrt{G_{H_{1}}}}\left(S^{(l)} \tilde{U}^{(l)} Y^{(e)} U^{(e)} S^{(e)}\right)_{I J}
\end{aligned}
$$

We use here the notation " " for transpose. In Eq. (30), $\lambda^{(u, d, e)}$ play the role of the Yukawa matrices at the GUT scale in the phenomenological analyses such as in [2]. However, in general they are not symmetric matrices and so M-Theory textures are uniquely different from what has previously been considered in phenomenological analyses. In brief, it is the smallness of the third generation eigenvalues of the Kahler matrices appearing in the denominators of Eq.(31)(33) (from the factor S of Eq.(24) ) that give rise to the large third generation masses.

\footnotetext{
${ }^{2}$ We correct an error in [28], the omission of the $V^{-\frac{1}{3}}$ factor in Eq. (13) (see e.g. 38]), which leads to a factor $1 / V^{-\frac{1}{2}}$ in Eq. 3133) rather then $1 / V$.
} 
Table 2. Kahler matrices $Z_{I J}^{(u, d, l, e)}$ and Yukawa matrices $Y^{(u, d, e)}$ for $\tan \beta=40$.

$$
\begin{gathered}
Z^{u}=f_{T}\left(\begin{array}{ccc}
1 & 0.3452 & 0 \\
0.3452 & 0.1311 & 0.006365 \\
0 & 0.006365 & 0.00344
\end{array}\right) ; Z^{d}=f_{T}\left(\begin{array}{ccc}
1 & 0.496 & 0 \\
0.496 & 0.564 & 0.435 \\
0 & 0.435 & 0.729
\end{array}\right) ; \\
Z^{l}=f_{T}\left(\begin{array}{ccc}
1 & -0.547 & 0 \\
-0.547 & 0.432 & 0.025 \\
0 & 0.025 & 0.09
\end{array}\right) ; Z^{e}=f_{T}\left(\begin{array}{ccc}
1 & 0.624 & 0 \\
0.624 & 0.397 & 0.00574 \\
0 & 0.00574 & 0.004407
\end{array}\right) \\
\operatorname{diag} Y^{(u)}=(0.0114,0.0597,0.104 \exp [0.65 \pi i]) ; \\
\operatorname{diag} Y^{(d)}=(2.052,0.2565,1.8297) ; \\
\operatorname{diag} Y^{(e)}=(0.307,3.789,1.821) .
\end{gathered}
$$

In [28] we saw for the case of $\tan \beta=3$ how the above Yukawa matrices gave rise to the experimental quark masses and CKM matrix elements at the electroweak scale, and we showed there analytically how the hierarchies arose naturally without undue fine tuning. We now update this analysis for the case of $\tan \beta=40$, and extend the discussion to include the lepton sector. Table 2 shows a choice of Kahler metric and Yukawa matrices that satisfy all the current experimental data. The $Z_{23}^{F}, Z_{32}^{F}$ and $Z_{33}^{F}$ entries for $F=u, l, e$ are $O\left(d^{2}\right)$ (for $d=0.1$ ) as required by Eq.(22). For simplicity we have assumed that the $q$ and $u$ quarks have identical Kahler matrices and have the maximum number of zero entries, and that the Yukawa matrices are diagonal. One phase is assumed in the Yukawa matrices to account for CP violation. To compare with low energy data, we use one loop Yukawa RGEs and two loop gauge RGEs to evaluate the Yukawa couplings at the electroweak scale, which we take to be $m_{t}$. Below $m_{t}$ we assume that the Standard Model holds and include in our calculations the QCD corrections (which are quite significant). The QCD correction factors used were $\eta_{c}=2, \eta_{u}=2.5=\eta_{d}, \eta_{b}=1.6$ and $\eta_{s}=2.5$. Diagonalization of the low energy Yukawa matrices then allows one to generate the low energy quark and lepton masses and the CKM matrix elements. The results are shown in Table 3, and are in good agreement with experiment. Of course in a fundamental analysis, the precise entries in 
Table 3. Quarks and leptons masses and CKM matrix elements obtained from the model of Table 2. Masses are in GeV. Experimental values for lepton and quark masses are from [32] and CKM entries from [39] unless otherwise noted.

\begin{tabular}{|c|c|c|}
\hline Quantity & Theoretical Value & Experimental Value \\
\hline$m_{t}($ pole $)$ & 175.2 & $174.3 \pm 5.1$ \\
$m_{c}\left(m_{c}\right)$ & 1.27 & $1.0-1.4$ \\
$m_{u}(1 \mathrm{GeV})$ & 0.00326 & $0.002-0.006$ \\
$m_{b}\left(m_{b}\right)$ & 4.21 & $4.0-4.5$ \\
$m_{s}(1 \mathrm{GeV})$ & 0.086 & $0.108-0.209$ \\
$m_{d}(1 \mathrm{GeV})$ & 0.00627 & $0.006-0.012$ \\
$m_{\tau}$ & 1.78 & 1.777 \\
$m_{\mu}$ & 0.1054 & 0.1056 \\
$m_{e}$ & 0.000512 & 0.000511 \\
$\left|V_{u s}\right|$ & 0.221 & $0.2210 \pm 0.0023$ \\
$\left|V_{c b}\right|$ & 0.042 & $0.0415 \pm 0.0011$ \\
$\left|V_{u b}\right|$ & $4.96 \times 10^{-3}$ & $3.80_{-0.13}^{+0.24} \pm 0.45 \times 10^{-3}$ \\
$\left|V_{t d}\right|$ & $6 \times 10^{-3}$ & $9.2 \pm 1.4 \pm 0.5 \times 10^{-3}$ \\
$\sin 2 \beta$ & 0.803 & $0.731 \pm 0.056[40]$ \\
\hline
\end{tabular}

Table 2 arise from integrals over the Calabi-Yau space, an analysis that cannot at this stage be performed. However, our discussion has shown that the general structure of the Kahler metric and Yukawa couplings arising in our Horava-Witten model can lead to low energy quark and lepton spectra consistent with all current experiments without the fine tuning used in phenomenological analyses.

Without knowledge of the value of the factors $R^{3} V^{1 / 2} \sqrt{G_{H_{1,2}}}$ in the denominators of Eq.(31]33), Kahler textures can only determine the mass ratios. As in 28], we use the top Yukawa at the GUT scale to determine the value of this common factor. If we write $V=r^{6}$, where $\mathrm{r}$ is the mean radius of the Calabi-Yau manifold divided by the co-ordinate radius, 
then for $G_{H_{1,2}}=1$, one finds that

$$
R \times r=6.82
$$

In the next section, we will show that $R$ and $r$ can be determined separately if massive neutrinos enter our model via the mechanism proposed there.

\section{NEUTRINO MASSES AND OSCILLATIONS}

In the last section we presented a way to generate the Yukawa textures in the quark and lepton sectors whose structures are the same as the SM. The consequence of the masslessness of neutrinos in the SM is that the mass eigenstates of leptons are identical to their gauge or flavor eigenstates and, unlike the quark sector which has a CKM mixing matrix, the lepton sector does not. Therefore, there is no oscillations between neutrinos in the SM. However, the neutrino experiments of Super-Kamiokande [41, 42], SNO [43] and KamLAND [44] have shown the existence of neutrino oscillations which indicates that neutrinos are actually massive particles. In this section we will show that massive neutrinos can be included in our model and their masses and mixings can be fitted into the large mixing angle (LMA) solution. (For a recent review of neutrino oscillations see [45].)

The simplest way to include massive neutrinos to our model is to associate a right-handed neutrino to every left-handed neutrino and insert by hand a term proportional to

$$
Y^{(\nu)} l_{L} H_{2} \nu_{R}
$$

into superpotential (29). However, the Yukawa couplings in the neutrino sector have to be extremely small and thus this solution is theoretically less interesting unless there is a mechanism behind it. The most widely used way to overcome this problem is the seesaw mechanism [29]. In seesaw models, besides the usual Dirac mass terms (which are approximately the same size as other fermion masses), one introduces additional very large Majorana masses which enter in the off-diagonal entries of the neutrino mass matrix. As a consequence, some eigenvalues are suppressed to the desired values when the diagonalization of neutrino mass matrix takes place. The physical neutrinos in seesaw models are then of Majorana type while other leptons and quarks are Dirac fermions. Here we propose a new way to generate neutrino masses. In our model, neutrinos are of Dirac type and thus the similarity between leptons and quarks is preserved and no neutrinoless double beta decay 
exists. We will see that our new mechanism provides a reasonable physical explanation to the origin of term (35).

The Kahler potential in principle can have gravitationally coupled trilinear terms which are usually ignored as they generally are of negligible size. However, we assume here that our Kahler potential at the GUT scale contains the holomorphic cubic term $K^{(3)}=K_{\nu}+K_{\nu}^{\dagger}$ where

$$
K_{\nu}=\kappa_{11} Y^{(\nu)} l_{L} H_{2} \nu_{R}
$$

where $1 / \kappa_{11}$ is the 11 dimensional Planck mass (i.e. $1 / \kappa_{11} \simeq M_{G}$ ) and $Y^{\nu}$ is a Yukawa matrix. We note that Eq.(36) is the only gauge invariant holomorphic cubic lepton term involving $\nu_{R}$ and that $\kappa_{11}$ is the natural scale for Horava-Witten theory. We assume here that the Yukawa contribution to the superpotential is still given by (30), and that no additional neutrino masses arise there. One can transfer $K_{\nu}$ from the Kahler potential to the superpotential by a Kahler transformation $\left(1 / \kappa_{4}\right.$ is the $4 \mathrm{D}$ Planck mass):

$$
\begin{aligned}
K & \rightarrow K-K^{(3)} \\
W & \rightarrow e^{\kappa_{4}^{2} K_{\nu}} W=W+\kappa_{4}^{2} K_{\nu} W+\cdots
\end{aligned}
$$

Now when supersymmetry breaks, the superpotential W will grow a VEV of size:

$$
\langle W\rangle \cong \frac{1}{\kappa_{4}^{2}} M_{S}
$$

where $M_{S}$ is of electroweak size. Consequently, after supersymmetry breaking, an additional term appears in superpotential (30):

$$
\frac{M_{S}}{M_{G}} Y^{(\nu)} l_{L} H_{2} \nu_{R}
$$

In the above we have assumed that no additional neutrino masses arise in the superpotential. One might imagine that this could come about if there exists a global symmetry or a non-gauge discrete symmetry that $\nu_{R}$ obeys. (Examples might be if $\nu_{R}$ was a member or a global $S U(2)_{R}$ doublet or were charged under a global $U(1)$ symmetry; or if $\nu_{R}$ appeared in $W$ only as an even power one could assign it a discrete quantum number -1 .) Then in the cubic part of the superpotential, the global or discrete symmetry (plus $S U(2)_{L}$ symmetry) would forbid Majorana or Dirac masses formed from $\nu_{L, R}$. Since gravity is expected to break such global or discrete symmetries in string theories, quartic terms scaled by $\kappa_{11}$ could arise 
(which is in fact why the contribution of Eq. (36) would be allowed in the Kahler potential in this scenario.) Such terms would have the general form $\left(H_{2} l\right)^{2}$ and $H_{1} H_{2} \nu_{R}^{2}$, giving rise to Majorana masses. Using the parameters of Table 2 and Eq. (43) below, we estimate that the first term gives rise to neutrino masses a factor of $\sim 100$ smaller than Eq. (36), and the second term is a factor of $1 / \tan \beta$ smaller yet. Thus they would produce only small corrections in our model. We have not investigated in detail if one could dispense with Eq. (36) completely and try to get the correct neutrino masses using only the above superpotential terms, but because of the above result, we believe it to be unlikely. We note also, that the above superpotential terms could also occur in the standard see-saw model, but are neglected there. While global and discrete symmetries can indeed arise from Calabi-Yau manifolds in Horava-Witted M-theory, whether or not the necessary symmetries are present in physically interesting Calabi-Yau manifolds is not known. Thus we have postulated their existence in this paper. ${ }^{3}$

We can now proceed as in Sec. 2. First diagonalize and rescale the Kahler matrices $Z_{I J}$ of $\nu_{R}$ and other fields to the unit matrix. Then make the necessary transformations in the superpotential to the canonical normalized fields. The term giving rise to neutrino masses can then be written as

$$
\nu_{L}^{\prime} \lambda^{(\nu)} \nu_{R}^{\prime} H_{2}^{\prime}
$$

where

$$
\lambda_{I J}^{(\nu)}=\frac{1}{\sqrt{2}} \frac{1}{R^{3 / 2}} \frac{1}{\sqrt{G_{H_{2}}}} \frac{M_{S}}{M_{G}}\left(S^{(l)} \tilde{U}^{(l)} Y^{(\nu)} U^{(\nu)} S^{(\nu)}\right)_{I J}
$$

Note that the overall coefficient in (41) is different from the one in (31]33) because the neutrino term originates from the Kahler potential, not the superpotential (27) which has the additional coefficient $e^{\frac{1}{2} K_{m}}$. It is thus possible to use the experimental neutrino mass square differences to determine $R$. In the example given below, we find that $R=2.13$ produces acceptable neutrino masses (we assume $M_{S}=1 \mathrm{TeV}$ in our calculation), and from Eq.(34), one finds that $r=3.20$. At the weak scale, after the diagonalization of charged lepton and neutrino Yukawa matrices, the Maki-Nakagawa-Sakata (MNS) lepton mixing matrix arises. We follow the standard parameterization [32] (the phase similar to the one

\footnotetext{
${ }^{3}$ We thank the Referee for bring this point to our attention.
} 
in the CKM matrix is ignored):

$$
V_{M N S}=\left(\begin{array}{ccc}
c_{12} c_{13} & s_{12} c_{13} & s_{13} \\
-s_{12} c_{23}-c_{12} s_{23} s_{13} & c_{12} c_{23}-s_{12} s_{23} s_{13} & s_{23} c_{13} \\
s_{12} s_{23}-c_{12} c_{23} s_{13} & -c_{12} s_{23}-s_{12} c_{23} s_{13} & c_{23} c_{13}
\end{array}\right) .
$$

where $c_{i j}=\cos \theta_{i j}, s_{i j}=\sin \theta_{i j}$ and $i, j=1,2,3$.

The following is an example at $\tan \beta=40$. We use the lepton entries of Table 2 , and the following neutrino Kahler and Yukawa matrices at $M_{G}$ :

$$
\begin{gathered}
Z^{\nu}=f_{T}\left(\begin{array}{ccc}
1 & -0.465 & 0 \\
-0.465 & 0.3105 & 0.0254 \\
0 & 0.0254 & 0.027
\end{array}\right) ; \\
\operatorname{diag} Y^{(\nu)}=(4,0.4,4) .
\end{gathered}
$$

The neutrino mass square differences and mixing angles at the weak scale are then calculated to be:

$$
\begin{gathered}
\Delta m_{21}^{2}=5.5 \times 10^{(-5)} \mathrm{eV}^{2} \\
\Delta m_{32}^{2}=2.7 \times 10^{(-3)} \mathrm{eV}^{2} \\
\tan ^{2} \theta_{12}=0.42 ; \tan ^{2} \theta_{23}=0.93 .
\end{gathered}
$$

with $\left|U_{e 3}\right|=0.005$. Since our model is a complete model of neutrino masses, we can calculate all the masses themselves and not just the mass square differences. For the above example we find

$$
m_{1}=6.5 \times 10^{-4} \mathrm{eV} ; \quad m_{2}=7.4 \times 10^{-3} \mathrm{eV} ; \quad m_{3}=5.2 \times 10^{-2} \mathrm{eV}
$$

consistent with cosmological constraints on neutrino masses [47].

The analysis of solar and KamLAND data in terms of two neutrino oscillations gives for the LMA solution [42]:

$$
0.20 \leq \tan ^{2} \theta_{S} \leq 0.68 ; \quad 5.6 \times 10^{-5} \leq \Delta m_{S}^{2} / \mathrm{eV}^{2} \leq 8.9 \times 10^{-5}
$$


where $\Delta m_{S}^{2}$ is the solar neutrino mass square difference and $\theta_{S}$ is the corresponding mixing angle and the ranges in Eq.(49) (and Eq.(50) below) are $3 \sigma$ around the central value. The analysis of Super-Kamiokande and K2K data shows for the LMA solution [48]:

$$
0.85 \leq \sin ^{2} 2 \theta_{A} \leq 1 ; \quad 1.4 \times 10^{-3} \leq \Delta m_{A}^{2} / \mathrm{eV}^{2} \leq 3.8 \times 10^{-3}
$$

where $\Delta m_{A}^{2}$ and $\theta_{A}$ are the relevant mass square difference and mixing angle for the atmospheric neutrino oscillation.

Since in our case $\left|U_{e 3}\right| \cong 0$, solar and atmospheric neutrino oscillations decouple [31, 49]. Therefore the two neutrino oscillation analysis can be applied to our case with the effective mixing angles given by:

$$
\theta_{S}=\theta_{12}, \quad \theta_{A}=\theta_{23}
$$

Eq.(45)-(47), (49) and (50) show that our results agree with the current LMA solution quite well.

\section{V. $\mu \rightarrow e+\gamma$ DECAY}

Lepton flavor violation (LFV) processes in supersymmetric models have been discussed in much detail in the literature (e.g. [50, 51, 52, 53, 54]). In our model the MNS matrix by itself can give rise to LFV processes, but this contribution is still very small, e.g. for $\mu \rightarrow e+\gamma$, the decay rate is of $\mathcal{O}\left(\left(m_{\nu} / m_{W}\right)^{4}\right)$ [55, 56, 57, 58]. Therefore, In this section, we mainly discuss the additional supersymmetric contributions to the LFV process $\mu \rightarrow e+\gamma$.

The operator for $\mu \rightarrow e+\gamma$ is:

$$
\mathcal{L}_{\mu \rightarrow e+\gamma}=\frac{i e}{2 m_{\mu}} \bar{e} \sigma^{\mu \nu} q_{\nu}\left(a_{l} P_{L}+a_{r} P_{R}\right) \mu \cdot A_{\mu}+\text { h.c. }
$$

where $P_{L, R} \equiv\left(1 \mp \gamma_{5}\right) / 2$ and $\sigma^{\mu \nu} \equiv \frac{i}{2}\left[\gamma^{\mu}, \gamma^{\nu}\right]$. The decay width for $\mu \rightarrow e+\gamma$ can be written as:

$$
\Gamma(\mu \rightarrow e+\gamma)=\frac{m_{\mu} e^{2}}{64 \pi}\left(\left|a_{l}\right|^{2}+\left|a_{r}\right|^{2}\right)
$$

Then the branching ratio is given by:

$$
\begin{aligned}
\operatorname{Br}(\mu \rightarrow e+\gamma) & \cong \frac{\Gamma(\mu \rightarrow e+\gamma)}{\Gamma\left(\mu \rightarrow e \bar{\nu}_{e} \nu_{\mu}\right)} \\
& =\frac{3 \pi^{2} e^{2}}{G_{F}^{2} m_{\mu}^{4}}\left(\left|a_{l}\right|^{2}+\left|a_{r}\right|^{2}\right)
\end{aligned}
$$



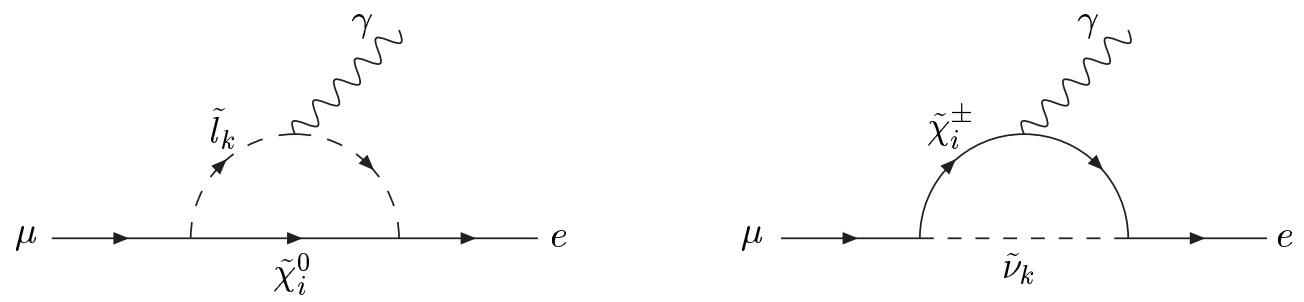

FIG. 1: Feynman diagrams for the neutralino and chargino contributions to $\mu \rightarrow e+\gamma$.

The supersymmetric contributions include the neutralino and chargino diagrams shown in FIG[1. The neutralino diagram gives:

$$
\begin{aligned}
& a_{l}^{N}=\sum_{i=1}^{4} \sum_{k=1}^{6} \frac{m_{\mu}^{2}}{8 \pi^{2} m_{\tilde{l}_{k}}^{2}}\left(N_{1 L}^{k i *} N_{2 L}^{k i} \mathrm{~F}_{1}\left(x_{i k}^{N}\right)+\frac{m_{\tilde{\chi}_{i}^{0}}}{m_{\mu}} N_{1 L}^{k i *} N_{2 R}^{k i} \mathrm{~F}_{2}\left(x_{i k}^{N}\right)\right) \\
& a_{r}^{N}=\sum_{i=1}^{4} \sum_{k=1}^{6} \frac{m_{\mu}^{2}}{8 \pi^{2} m_{\tilde{l}_{k}}^{2}}\left(N_{1 R}^{k i *} N_{2 R}^{k i} \mathrm{~F}_{1}\left(x_{i k}^{N}\right)+\frac{m_{\tilde{\chi}_{i}^{0}}}{m_{\mu}} N_{1 R}^{k i *} N_{2 L}^{k i} \mathrm{~F}_{2}\left(x_{i k}^{N}\right)\right)
\end{aligned}
$$

where $x_{i k}^{N}=m_{\tilde{\chi}_{i}^{0}}^{2} / m_{\tilde{l}_{k}}^{2}$ and

$$
\begin{aligned}
\mathrm{F}_{1}(x) & =\frac{2 x^{3}+3 x^{2}-6 x+1-6 x \ln x}{12(x-1)^{4}} \\
\mathrm{~F}_{2}(x) & =\frac{x^{2}-1-2 x \ln x}{2(x-1)^{3}} \\
N_{l L}^{k i} & =\sqrt{2} g_{1} X_{i 1} D_{l+3, k}^{*}+X_{i 3} D_{l k}^{*} Y_{l}^{e(D)} \\
N_{l R}^{k i} & =-\frac{1}{\sqrt{2}}\left(g_{1} X_{i 1}+g_{2} X_{i 2}\right) D_{l k}^{*}+X_{i 3} D_{l+3, k}^{*} Y_{l}^{e(D)}
\end{aligned}
$$

where $g_{1}$ and $g_{2}$ are the $\mathrm{U}(1)$ and $\mathrm{SU}(2)$ gauge coupling constants, $X$ is the matrix diagonalizing the $4 \times 4$ neutralino mass matrix $M_{\tilde{\chi}^{0}}$ according to $M_{\tilde{\chi}^{0}} X=X^{*} M_{\tilde{\chi}^{0}}^{(D)}, D$ diagonalizes the $6 \times 6$ charged slepton mass matrix $M_{\tilde{l}}^{2}$ according to $M_{\tilde{l}}^{2} D=D M_{\tilde{l}}^{2(D)}$ and $Y^{e(D)}$ is the diagonalized Yukawa matrix of charged leptons (We use the notation of [59]).

Similarly, the chargino diagram gives

$$
\begin{aligned}
& a_{l}^{C}=-\sum_{i=1}^{2} \sum_{k=1}^{6} \sum_{m=1}^{3} \frac{m_{\mu}^{2}}{8 \pi^{2} m_{\tilde{\nu}_{k}}^{2}}\left(C_{1 L}^{k i}{ }^{*} C_{2 L}^{k i} \mathrm{~F}_{3}\left(x_{i k}^{C}\right)+\frac{m_{\tilde{\chi}_{i}^{ \pm}}}{m_{\mu}} C_{1 L}^{k i *} C_{2 R}^{k i} \mathrm{~F}_{4}\left(x_{i k}^{C}\right)\right) \\
& a_{r}^{C}=-\sum_{i=1}^{2} \sum_{k=1}^{6} \sum_{m=1}^{3} \frac{m_{\mu}^{2}}{8 \pi^{2} m_{\tilde{\nu}_{k}}^{2}}\left(C_{1 R}^{k i}{ }^{*} C_{2 R}^{k i} \mathrm{~F}_{3}\left(x_{i k}^{C}\right)+\frac{m_{\tilde{\chi}_{i}^{ \pm}}}{m_{\mu}} C_{1 R}^{k i *} C_{2 L}^{k i} \mathrm{~F}_{4}\left(x_{i k}^{C}\right)\right)
\end{aligned}
$$


where $x_{i k}^{C}=m_{\tilde{\chi}_{i}^{ \pm}}^{2} / m_{\tilde{\nu}_{k}}^{2}$ and

$$
\begin{aligned}
\mathrm{F}_{3}(x) & =\frac{x^{3}-6 x^{2}+3 x+2+6 x \ln x}{12(x-1)^{4}} \\
\mathrm{~F}_{4}(x) & =\frac{x^{2}-4 x+3+2 \ln x}{2(x-1)^{3}} \\
C_{l L}^{k i} & =-g_{2} V_{i 1} P_{m k}^{*}\left(V_{M N S}\right)_{l m}^{*}+V_{i 2} P_{m+3, k}^{*} Y_{m}^{\nu(D)}\left(V_{M N S}\right)_{l m}^{*} \\
C_{l R}^{k i} & =U_{i 2}^{*} P_{m k}^{*}\left(V_{M N S}\right)_{l m}^{*} Y_{l}^{e(D)}
\end{aligned}
$$

where $V_{M N S}$ is the MNS mixing matrix, $P$ is the matrix diagonalizing the $6 \times 6$ sneutrino mass matrix $M_{\tilde{\nu}}^{2}$ according to $M_{\tilde{\nu}}^{2} P=P M_{\tilde{\nu}}^{2(D)}, U$ and $V$ diagonalize the chargino mass matrix $M_{\tilde{\chi}^{ \pm}}$according to $U^{*} M_{\tilde{\chi}^{ \pm}} V^{\dagger}=M_{\tilde{\chi}^{ \pm}}^{(D)}$ and $Y^{\nu(D)}$ is the diagonalized Yukawa matrix of neutrinos.

To evaluate the branching ratio of $\mu \rightarrow e+\gamma$, we first generate Yukawa textures in the way described in Sec. 3 with phenomenological inputs including the fermion masses and neutrino oscillations described in Sec. 4. Then we choose soft breaking parameters at the GUT scale and run the RGEs to the weak scale. Finally one can use the formula given above to calculate the $\mu \rightarrow e+\gamma$ branching ratio. We display our results in the following three figures for $\tan \beta=10,30$ and 40 .

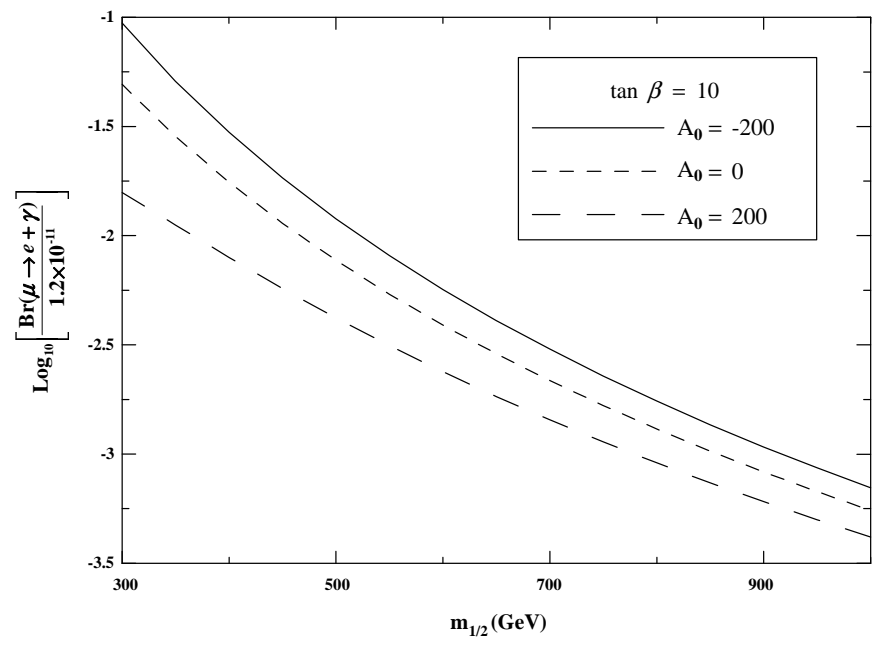

FIG. 2: Branching ratio of $\mu \rightarrow e+\gamma$ for $\tan \beta=10$

Although our Yukawa textures are constructed through the Kahler potential, the usual mSUGRA structure holds at the GUT scale. In addition, to constrain the parameter space, 


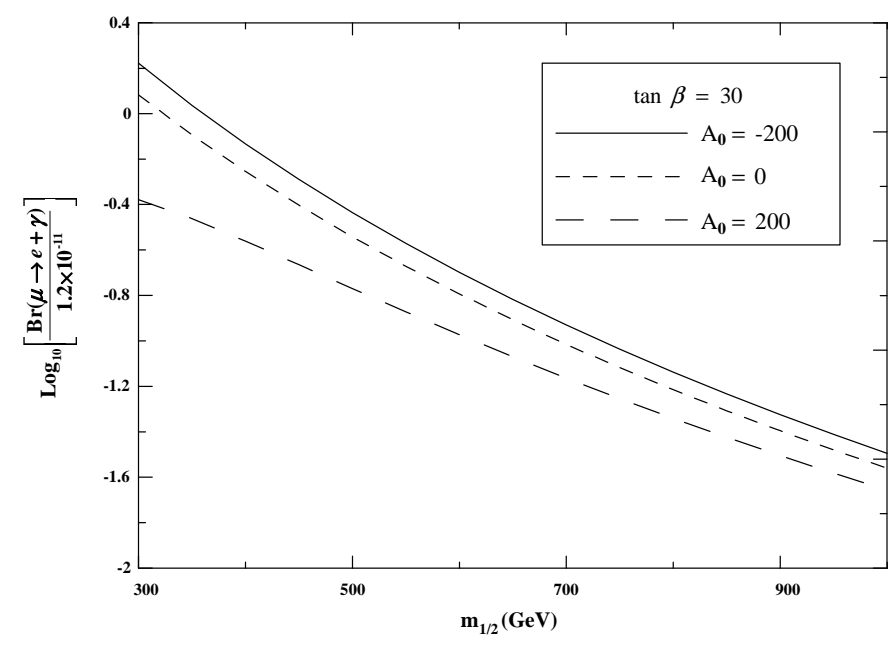

FIG. 3: Branching ratio of $\mu \rightarrow e+\gamma$ for $\tan \beta=30$

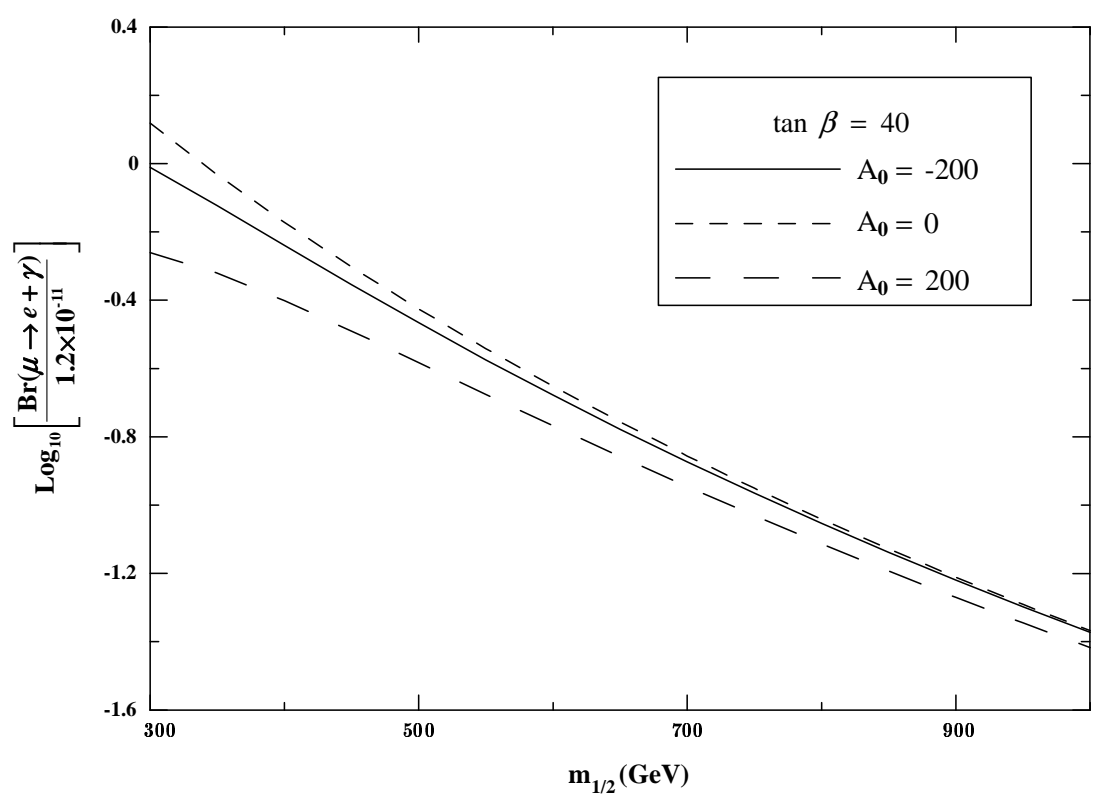

FIG. 4: Branching ratio of $\mu \rightarrow e+\gamma$ for $\tan \beta=40$

we implement the relic density constraint [47]: $0.095 \leq \Omega_{\chi_{1}^{0}} h^{2} \leq 0.129$. For a given $m_{1 / 2}$, an allowed narrow region of $m_{0}$ is determined by the relic density constraint [59, 60]. Since the $\mu \rightarrow e+\gamma$ branching ratio is not sensitive to the value of $m_{0}$ we only show the result for one value of $m_{0}$ in the allowed region for any given $m_{1 / 2}$. There are other experimental bounds on the parameter space, e.g. the $b \rightarrow s+\gamma$ decay, the light Higgs mass, the muon $g-2$, all of which can easily be implemented. Since $m_{0}$ is significantly constrained by the relic density 
bounds for any given $m_{1 / 2}$, other experimental constraints are only needed for constraining $m_{1 / 2}$. For example, at $\tan \beta=40$, the $b \rightarrow s+\gamma$ branching ratio produces a lower bound of $\simeq 400 \mathrm{GeV}$ on $m_{1 / 2}$ while a muon $g-2$ deviation from the SM can produce an upper bound on $m_{1 / 2}$. For the purpose of showing what $\mu \rightarrow e+\gamma$ branching ratio can be reached in our model, except for the relic density constraint, we ignore the other experimental bounds in our plots since they are not significant for this purpose.

In our plots the y-axis is the logarithmic ratio of our theoretical predictions to the current experimental bound [32]. Therefore, only the region below zero is experimentally allowed. One can see that at large $\tan \beta$, especially $\operatorname{for} \tan \beta=40$, the theory predictions are only about one order of magnitude smaller than the experimental bound and hence accessible to future experiments [33, 34] while part of the parameter space for lower $\tan \beta$ will also be accessible.

\section{CONCLUSION}

In this paper we have extended a model of the quark mass hierarchy based on the Horava-Witten M-Theory [28] to include charged leptons and massive neutrinos. The model is based on the assumptions that five branes exists in the bulk lying near the distant orbifold plane (i. e. about $90 \%$ of the way from the physical plane), and that the instanton charges on the physical plane vanish. We had previously seen that this gave rise to a three generation model with the Standard Model gauge group at the GUT scale. While one cannot calculate Yukawa couplings in M-Theory (they involve integrals over the Calabi-Yau space) these constraints were sufficient to qualitatively account for the quark mass hierarchy at the electroweak scale without undue fine tuning. The mechanism that achieved this was that the five brane contribution to the Kahler potential gave rise to small Kahler matrix eigenvalues, and the quark masses were proportional to the reciprocal square root of the eigenvalues when the kinetic energy was put into canonical form. We saw that the same mechanism also gave rise qualitatively to the hierarchy of charged lepton masses, again without any excessive fine tuning.

Neutrino masses can arise in these models if a right handed neutrino exists in the massless particle spectrum. Then one can assume that the Kahler potential has a cubic holomorphic contribution of the form of Eq.(36), the interaction being scaled by the 11 dimensional 
Planck mass (the basic parameter of Horava-Witten theory). When transformed to the superpotential by a Kahler transformation, this term gives rise to neutrino masses of the correct size after supersymmetry breaking. (Thus the mechanism being used here for the neutrino masses is similar to the one previously used to generate a $\mu$ parameter of electroweak size [61].) it is possible then to chose natural sized values for the Yukawa and Kahler matrix entries to generate masses and CKM and MNS mixing angles in agreement with all low energy data. The neutrinos in this model are Dirac, and so will exclude neutrinoless double beta decay. However, the mixing in the neutrino sector allows for $\mu \rightarrow e+\gamma$ decay to occur, and with reasonable values of the SUSY parameters, this decay should become observable in the next round of $\mu$ catalysis experiments [33, 34] over a significant range of parameters.

Aside from the Kahler and Yukawa matrices, the quark, lepton and neutrino properties depend on the Calabi-Yau volume modulus $\mathcal{V}$ which we have parameterized by $V^{1 / 6}=r$ and the radius modulus $\mathrm{R}$. We have found that all the quark, lepton and neutrino masses can be fit satisfactorily with $r$ and $R$ of $O(1)$. Thus for the example in text for $\tan \beta=40$ we found $R=2.13$ and $r=3.20$. One important feature of this Horava-Witten model that has not been addressed here is how to stabilize the position of the 5-brane close to the distant orbifold plane. One possibility may involve quantum corrections, e.g. membrane potentials between the 5 -brane and the orbifold planes [38, 62, 63].

\section{Acknowledgments}

This work was supported in part by National Science Foundation Grant PHY-0101015 and in part by the Natural Science and Engineering Research Council of Canada.

[1] H. Georgi and C. Jarlskog, Phys. Lett. B86, 297 (1979).

[2] P. Ramond, R. G. Roberts and G. G. Ross, Nucl. Phys. B406, 19 (1993) hep-ph/9303320.

[3] P. Horava and E. Witten, Nucl. Phys. B460 506 (1996) hep-th/9510209.

[4] P. Horava and E. Witten, Nucl. Phys. B475 94 (1996) hep-th/9603142.

[5] E. Witten, Nucl. Phys. B471 135 (1996) hep-th/9602070.

[6] P. Horava, Phys. Rev. D 547561 (1996) hep-th/9608019.

[7] T. Banks and M. Dine, Nucl. Phys. B479 173 (1996) hep-th/9605136. 
[8] R. Friedman, J. Morgan and E. Witten, Commun. Math. Phys.187 679 (1997) hep-th/9701162.

[9] R. Friedman, J. Morgan and E. Witten, alg-geom/9709029.

[10] R. Donagi, Asian J. Math. 1 214(1997).

[11] A. Lukas, B. Ovrut and D. Waldram, Nucl. Phys. B532 43 (1998) hep-th/9710208.

[12] A. Lukas, B. Ovrut and D. Waldram, Phys. Rev. D 577529 (1998) hep-th/9711197.

[13] B. Andreas, JHEP 9901, 011 (1999) hep-th/9802202.

[14] G. Curio,Phys. Lett. B 435391 (1998) hep-th/9803224.

[15] A. Lukas, B. Ovrut, K.S. Stelle and D. Waldram, Nucl. Phys. B552 246 (1999) hep-th/9806051.

[16] Z. Lalak, S. Pokorski and S. Thomas, Nucl. Phys. B549 63 (1999) hep-ph/9807503.

[17] A. Lukas, B. Ovrut and D. Waldram, Phys. Rev. D 59106005 (1999) hep-th/9808101.

[18] R. Donagi, A. Lukas, B. Ovrut and D. Waldram, JHEP 9905018 (1998) hep-th/9811168.

[19] R. Donagi, A. Lukas, B. Ovrut and D. Waldram, JHEP 9906034 (1999) hep-th/9901009.

[20] A. Lukas, B. Ovrut and D. Waldram, JHEP 9904009 (1999) hep-th/9901017.

[21] B. Ovrut, hep-th/9905115, Lectures at Asian Pacific Center for Theoretical Physics, Third Winter School, January 21-February 5, 1999, Cheju Island, Korea.

[22] A. Lukas and B. Ovrut, hep-th/9908100

[23] R. Donagi, B. Ovrut, T. Pantev and D. Waldram, Adv. Theor. Math. Phys. 5, 93 (2002) hep-th/9912208.

[24] M. Brandle, A. Lukas and B. Ovrut, Phys. Rev. D 63, 026003 (2001) hep-th/0003256.

[25] G. Curio and A. Krause, Nucl. Phys. B 602, 172 (2001) hep-th/0012152.

[26] B. Andreas and D. Hernandez-Ruiperez, hep-th/0305123.

[27] G. Curio and A. Krause, hep-th/0308202.

[28] R. Arnowitt and B. Dutta, Nucl. Phys. B592, 143 (2001) hep-th/0006172.

[29] M. Gell-Mann, P. Ramond and R. Slansky in Sanibel Talk, CALT-68-709, Feb 1979, and in Supergravity (North Holland, Amsterdam 1979); T. Yanagida in Proc. of the Workshop on Unified Theory and Baryon Number of the Universe, KEK, Japan, 1979; R. N. Mohapatra and G. Senjanovic, Phys. Rev. Lett. 44, 912 (1980).

[30] Z. Maki, M. Nakagawa and S. Sakata, Prog. Theor. Phys. 28, 870 (1962).

[31] M. C. Gonzalez-Garcia and C. Pena-Garay, hep-ph/0306001. 
[32] K. Hagiwara et al., Phys. Rev. D 66, 010001 (2002).

[33] T. Mori et al., Search for $\mu^{+} \rightarrow e^{+} \gamma$ down to $10^{-14}$ branching ratio, http://meg.web.psi.ch/docs/prop_psi/index.html, 1999.

[34] MECO and KOPIO collaborations, A proposal to the NSF to construct the MECO and KOPIO experiments, http://meco.ps.uci.edu/RSVP.html, 1999.

[35] R. Arnowitt, B. Dutta and B. Hu hep-ph/0211084.

[36] A. E. Faraggi, R. Garavuso and J. M. Isidro, arXiv:hep-th/0209245.

[37] A. E. Faraggi and R. S. Garavuso, Nucl. Phys. B 659, 224 (2003) hep-th/0301147.

[38] E. Lima, B. A. Ovrut, J. Park and R. Reinbacher, Nucl. Phys. B 614, 117 (2001) hep-th/0101049.

[39] K. Schubert, talk given at XXI international symposium on lepton and photon interactions at high energies, Fermilab, Batavia, Illinois USA (2003), http://conferences.fnal.gov/lp2003/program/S6/schubert_s06.pdf.

[40] Heavy Flavor Averaging Group, http://www.slac.stanford.edu/xorg/hfag/index.html.

[41] Super-Kamiokande Collaboration, S. Fukuda et al., Phys. Rev. Lett. 85, 3999-4003 (2000).

[42] Super-Kamiokande Collaboration, hep-ex/0309011.

[43] SNO collaboration, Q.R. Ahmad et al., Phys. Rev. Lett. 87, 071301 (2001). Phys.Rev.Lett. 89, 011301 (2002); Phys.Rev.Lett 89, 011302 (2002); nucl-ex/0204009.

[44] K. Eguchi et al. [KamLAND Collaboration], Phys. Rev. Lett. 90, 021802 (2003) hep-ex/0212021.

[45] S. M. Bilenky, hep-ph/0307186.

[46] V. Barger, M. Berger and P. Ohmann, Phys. Rev. D49 4908 (1994) hep-ph/9311269.

[47] D. N. Spergel et al., astro-ph/0302209.

[48] G. L. Fogli, E. Lisi, A. Marrone and D. Montanino, Phys. Rev. D 67, 093006 (2003) hep-ph/0303064.

[49] S. M. Bilenky and C. Giunti, Phys. Lett. B 444, 379 (1998).

[50] D. F. Carvalho, M. E. Gomez and J. C. Romao, Phys. Rev. D 65, 093013 (2002) hep-ph/0202054

[51] W. Porod and W. Majerotto, Phys. Rev. D 66, 015003 (2002) hep-ph/0201284

[52] Z. Chacko and G. D. Kribs, Phys. Rev. D 64, 075015 (2001) hep-ph/0104317

[53] S. w. Baek, T. Goto, Y. Okada and K. i. Okumura, Phys. Rev. D 64, 095001 (2001) 
hep-ph/0104146

[54] X. J. Bi, Y. B. Dai and X. Y. Qi, Phys. Rev. D 63, 096008 (2001) [Phys. Rev. D 66, 076006 (2002)] hep-ph/0010270.

[55] S. T. Petcov, Sov. J. Nucl. Phys. 25, 340 (1977), yad. Fiz. 25, 641 (1977), Errata: SJNP 25, 698 (1977) YF 25, 1336 (1977).

[56] W. J. Marciano and A. I. Sanda, Phys. Lett. B 67, 303 (1977).

[57] B. W. Lee, S. Pakvasa, R. E. Shrock, and H. Sugawara, Phys. Rev. Lett. 38, 937 (1977), erratum: 38, 1230 (1977).

[58] B. W. Lee and R. E. Shrock, Phys. Rev. D 16, 1444 (1977).

[59] R. Arnowitt, B. Dutta and Y. Santoso, Phys. Rev. D 64, 113010 (2001) hep-ph/0106089

[60] J. R. Ellis, T. Falk, G. Ganis, K. A. Olive and M. Srednicki, Phys. Lett. B 510, 236 (2001) hep-ph/0102098; M. E. Gomez and J. D. Vergados, Phys. Lett. B 512, 252 (2001) hep-ph/0012020; H. Baer, C. Balazs, A. Belyaev, J. K. Mizukoshi, X. Tata and Y. Wang, hep-ph/0210441; A. B. Lahanas and D. V. Nanopoulos, hep-ph/0303130.

[61] S. K. Soni and H. A. Weldon, Phys. Lett. B 126, 215 (1983); G. F. Giudice and A. Masiero, Phys. Lett. B 206, 480 (1988).

[62] G. W. Moore, G. Peradze and N. Saulina, Nucl. Phys. B 607, 117 (2001) hep-th/0012104.

[63] G. Curio and A. Krause, Nucl. Phys. B 643, 131 (2002) hep-th/0108220. 